\title{
Brain Tumor Segmentation and Stage Detection in Brain MR Images with 3D Assessment
}

\author{
Purnita Majumder \\ Research Scholar \\ Government College of Engineering \\ Aurangabad, India
}

\author{
V.P. Kshirsagar \\ Head of The Department \\ Government College of Engineering \\ Aurangabad, India
}

\begin{abstract}
This paper deals with the detection of tumor stage in brain MR images with efficient algorithm and 3D assessment of brain for tumor detection providing better result. Tumor is an abnormal growth of tissues reproducing themselves in any part of the body. There are different types of tumor having different Characteristics and treatment. A large number of people having brain tumors die due to inaccurate detection. Magnetic resonance imaging (MRI) is a diagnostic procedure that uses a combination of radio frequencies, large magnet, and a computer to generate detailed images of organs and structures within the body. MR image is examined visually by the physician for detection \& diagnosis of brain tumor. However this method of detection consists of less accuracy while detecting the stage \& size of tumor. This project uses a computer aided method for segmentation (detection) of brain tumor based on the combination of algorithms. In this method segmentation of tumor tissue is done with accuracy and reproducibility than manual segmentation with less analysis time. then the tumor is extracted from the MR image and its exact position, shape and stage is determined. Then 3D analysis of brain MRI with the help of 3D analyzer tool and graph generation for tumor growth rate of particular patients and tumor types will be done.
\end{abstract}

\section{General Terms}

Segmentation

\section{Keywords}

3D Assessment, Brain tumor, Fuzzy C-means (FCM), Kmeans, Magnetic Resonance Imaging (MRI), Pre-processing

\section{INTRODUCTION}

This paper deals with automatic brain tumor segmentation and 3D assessment of brain. There are various ways to view the anatomy of the Brain such as MRI scan or CT scan. In CT the effective radiation dose ranges from 2 to $10 \mathrm{mSv}$. While an average person receives the same from background radiation in 3 to 5 years. For pregnant women or children CT is not recommended usually. The MRI scan is more comfortable than CT scan for diagnosis. In this paper we consider the MRI scanned image for the whole process. Among different types of algorithm for brain tumor detection, in this paper we used two algorithms for segmentation which gives the accurate result for tumor segmentation. More than 120 types of brain and central nervous system (CNS) tumors are existing. Almost all medical institutions use the World Health Organization (WHO) identification system to classify brain tumors. According to WHO brain tumors are classified by cell origin and the behavior of the cells, from the least aggressive (benign) to the most aggressive (malignant). Brain tumor normally affects CSF (Cerebral Spinal Fluid). It is the cause of strokes in the human body. So the patients get the treatment for the strokes instead of the treatment for tumors. So accurate detection of the tumor is important phase of the treatment.
The lifespan of a brain tumor affected person can be increased if it is detected at current stage. It will help to maximize the lifetime by 1 to 2 years. Among the two types of tumor cells the malignant tumor detection is somewhat more difficult than a mass tumor. The accurate detection of the malignant tumor needs a 3-D representation of brain and 3-D analyzer tool. In this paper we focused on detection of both mass and malignant tumor detection. We use MATLAB as the developing platform for the detection of tumor. Then we are providing systems that detect the tumor, its shape and the stage of a particular type of tumor. At the end we will go for 3D analysis of brain of tumor affected persons which will be helpful for the doctors and for the researchers [1].

\section{LITERATURE SURVEY}

This section consists of brief descriptions of few image encryption methods.

\subsection{Segmentation of Brain Tumor and Its} Area Calculation in Brain MR Images using K-Mean Clustering and Fuzzy CMean Algorithm

The method was proposed by J.selvakumar, A.Lakshmi, and T.Arivoli.This uses a method for brain tumor segmentation (detection) based on the combination of two algorithms. This allows the tumor tissue segmentation with accuracy and reproducibility as compared to manual segmentation. Time for analysis is also used. The tumor is extracted from the MR image and its exact position and the shape also determined at the end of the process. Based on the amount of area calculated from the cluster the stage of the tumor is displayed [1].

\subsection{An Adaptive Spatial Fuzzy Clustering} Algorithm for 3-D MR Image Segmentation This method was proposed by Alan Wee-Chung Liew and Hong Yan. In this paper an adaptive spatial fuzzy c-means clustering algorithm is used for the segmentation of threedimensional (3-D) magnetic resonance (MR) images. The input images may be corrupted by noise and intensity nonuniformity (INU) artifact. The efficacy of the proposed algorithm is demonstrated by extensive segmentation experiments using both simulated and real MR images and by comparison with other published algorithms [2]

\subsection{Separation of Brain Tissues in MRI} based on Multidimensional FCM and Spatial Information

This technique was proposed by Jamal Ghasemi, Mohamad Reza Karami mollaei and Ali Hojjatoleslami. A novel method for brain MRI segmentation (BMS) based on multidimensional standard FCM has been proposed. Different features of neighboring pixels like mean, singular value, and standard deviation in combination with pixel intensity has 
been used for typical pixel segmentation. The results evaluation is done against manual segmentation on a publicly available dataset [3].

\subsection{An Improved MR Image Segmentation Method Based on Fuzzy C-Means Clustering}

The technique was proposed by Linju Lu, Min Li. A novel algorithm, based on the k-nearest neighbor (KNN) algorithm and kernel fuzzy c-means (KFCM) clustering algorithm is used for the performance improvement of MR image segmentation. To speed up the algorithm the statistical gray level histogram of the image is used in KFCM algorithm. Based on kernel methods the spatial information of the image is also considered by k-nearest neighbor algorithm. With kernel methods each pixel of the image is mapped into a highdimensional feature space where the FCM algorithm and $\mathrm{KNN}$ algorithm are carried out. Experiments show that this is effective and efficient in image segmentation [4].

\subsection{Fuzzy Local Gaussian Mixture Model for Brain MR Image Segmentation}

The technique was proposed by Zexuan Ji, Yong Xia, Quansen Sun, Qiang Chen, Deshen Xia, and David Dagan Feng. In this paper, the author assumes that the local image data within each voxel's neighborhood satisfy the Gaussian mixture model (GMM), and thus propose the fuzzy local GMM (FLGMM) algorithm for automated brain MR image segmentation. The segmentation result that maximizes the posterior probability is estimated with this algorithm by minimizing an objective energy function, in that a truncated Gaussian kernel function is used to impose the spatial constraint and fuzzy memberships are employed to balance the contribution of each GMM. Author compared their algorithm to state-of-the-art segmentation approaches with synthetic and clinical data. The results of this technique show that it can largely overcome the issues raised by noise, bias field, low contrast, and substantially improve the accuracy of brain MR image segmentation [5].

\section{PROPOSED SYSTEM}

In this section, the main idea used in the proposed technique is described. The proposed system has mainly six modules: preprocessing, segmentation, Feature extraction, Stage detection, 3D assessment, and graph generation. In Pre processing filtering is performed on the MR image. Advanced K-means and Fuzzy C-means algorithm are used in segmentation work. Thresholding is used for feature extraction. To recognize the tumor shape, position and stage of tumor in MRI image edge detection method is used [1].

The proposed method consists of two algorithms. As shown the diagram below, the proposed system, is divided into the following 6 main phases:

The flow for proposed system is shown in Figure 1.

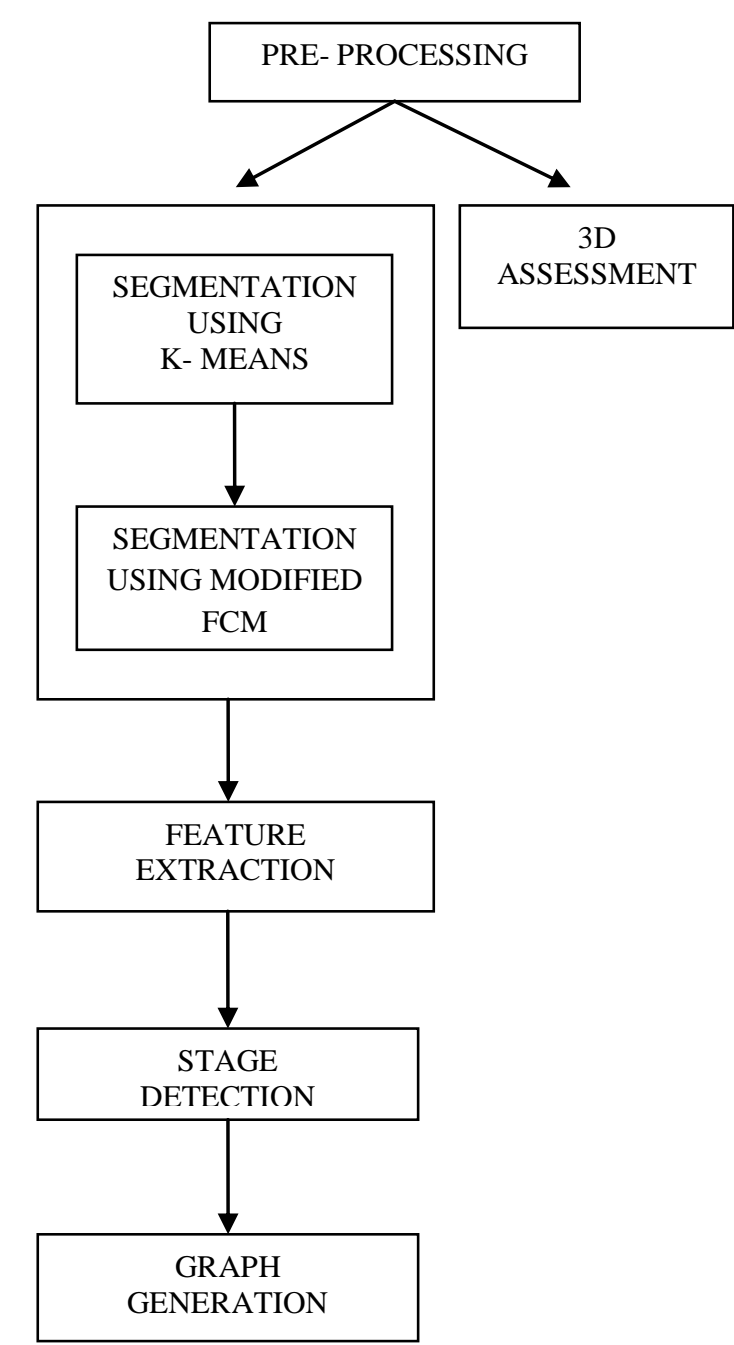

Fig 1: Flow Diagram for Proposed System

\subsection{PRE- PROCESSING}

In pre processing step the image is converted according to the need of the noise removal. Filtering of noise and other artifacts are performed in the image and edge sharpening is done in the image. RGB to grey conversion takes place followed by Reshaping. Median filter is used for noise removal. Noise arrival possibilities in modern MRI scan are very less but due to the thermal effect it may appear. The main aim of this paper is tumor detection, segmentation and $3 \mathrm{D}$ assessment. But for the better result it needs the process of noise removal.

\subsection{K-MEANS SEGMENTATION}

One of the unsupervised learning algorithms for clusters is Kmeans. Grouping the pixels according to some characteristics is called clustering the image. Initially we have to define the number of clusters $\mathrm{k}$ in the $\mathrm{k}$-means algorithm. Then randomly k-cluster center are chosen. The calculations of distance between each pixel to each cluster centers are done. That distance might be of simple Euclidean function. Using the distance formula all cluster centers are compared to Single pixel. Then the pixel is shifted to a particular cluster which is having the shortest distance among all. After that the centroid is re-estimated. Each pixel is compared to all centroids again and again. The process continuous in this way until the center converges. 

ALGORITHM
1. Give $\mathrm{k}$ as the no of cluster value.
2 . $\mathrm{k}$ cluster centers are chosen randomly.
3 . Calculate center or mean of the cluster.
4. Calculate the distance $\mathrm{b} / \mathrm{w}$ each cluster center to each pixel.
5. Move to that Cluster if the distance is near to the center.
6. Move to next cluster Otherwise.
7. Re-calculate the center.
8. Until the center doesn't move repeat the process.

\subsection{SEGMENTATION USING MODIFIED FUZZY C-MEAN CLUSTERING}

A way to processing the data by giving a partial membership value to each pixel of the image is fuzzy logic. The range of the membership value of the fuzzy set is 0 to 1. Fuzzy clustering is a multi valued logic which allows intermediate values i.e., in a same image member of one fuzzy set can also be a member of other fuzzy sets. No abrupt transition is there between non membership and full membership. The membership function is used to define the fuzziness of an image and the information contained in the image. These are three main basic features involved in characterized by membership function. They are boundary and support. A fully member of the fuzzy set is the core. The non membership value of the set is support and the intermediate or partial membership with value between 0 and 1 is boundary. In this work we will use modified FCM which will give more accuracy then the previous FCM clustering.

\subsection{FEATURE EXTRACTION}

The feature extraction is cluster extraction which shows the predicted tumor after MFCM. The extracted cluster from FCM output is given to the thresholding process as input. Binary mask is applied over the entire image. It makes the white become brighter and dark pixel become darker. Each transform coefficient is compared with a threshold in threshold coding. If transform coefficient is less than the threshold then it is considered as zero. If transform coefficient is larger than the threshold, it will be considered as one. Thresholding is an adaptive method in which only those coefficients having magnitudes above a threshold are retained within each block. Let us consider an image 'f having $\mathrm{k}$ gray level an integer value of $\mathrm{T}$ threshold, which lies in the $\mathrm{k}$ gray scale range. Thresholding is a comparison process. $T$ 'is compared to each pixel in 'f. Binary decision is made Based on that. Value of the particular pixel in an output binary image ' $\mathrm{g}$ ' is defined by that:

$$
\mathrm{g}(\mathrm{n})=\left\{\begin{array}{l}
0 \text { if } f(n) \geq T \\
1 \text { if } f(n)<T
\end{array}\right.
$$

\subsection{STAGE DETECTION}

Using the binarization method in the approximate reasoning step the area of the tumor is calculated. That means the image having two values either white or black ( 1 or 0$)$. Here maximum image size is $256 \times 256$ jpeg image. We can represent a binary image as a summation of total number of black and white pixels.

$$
\text { Image, } \mathrm{I}=\sum_{W=0}^{255} \sum_{H=0}^{255}[f(0)+f(1)]
$$

Pixels $=$ Height $(\mathrm{H}) \mathrm{X}$ Width $(\mathrm{W})=256$ X 256

$\mathrm{f}(\mathrm{l})=$ black pixel (digit 1$)$

$f(0)=$ white pixel $(\operatorname{digit} 0)$
No_of_white_pixel, $\mathrm{P}=\sum_{W=0}^{255} \sum_{H=0}^{255}[f(0)]$

Where,

$\mathrm{P}=$ Total number of white pixels (height $*$ width)

1 Pixel $=0.264 \mathrm{~mm}$

The formula for area calculation is

$$
\text { Size_of_tumor, } \mathrm{S}=[(\sqrt{P}) 0.264] \mathrm{mm}^{2}
$$

$\mathrm{P}=$ Number of white pixels; $\mathrm{H}=$ height, $\mathrm{W}=$ width;

\section{ALGORITHM}

The algorithmic steps involved for brain tumor shape detection is as follows-

Step 1: Start.

Step 2: Provide the MRI scan image in JPEG format as input. Step 3: Check the input image format is as specified or not and go to step 4, display error message if not in specified format.

Step 4: Covert image into gray scale image if it is in RGB format else move to next step.

Step 5: Find the grayscale image edge.

Step 6: Calculate the total number of white points in the image.

Step 7: Using the formula calculate the size of the tumor.

Step 8: Display the tumor size and stage.

Step 9: Stop the process.

The stage determination of tumor is based on the area of tumor. The proposed work uses techniques to calculate the tumor stage presented by J.selvakumar, A.Lakshmi, T.Arivoli in [1].

\subsection{GRAPH GENERATION}

Graphs will be generated to compare various features like tumor size, growth etc for a particular type of tumor and for a particular patient history so that it can be useful for the further treatment of the patient in future.

\subsection{D ASSESMENT}

It will be done with the help of 3D analyzer tool i.e. 3D slicer Matlab Bridge. With the help of this we can do analysis of Brain MRI which will be helpful for research purpose also.

\section{CONCLUSION}

Among different types of tumors available they may be exist as malignant over the brain or mass in brain. To extract mass tumor from brain cells MRI K- means algorithm is enough. If there is any noise present in the MR image then it is removed before the $\mathrm{K}$-means process. Input to the $\mathrm{k}$-means is the noise free image and tumor will be extraction will be done from the MRI image. And then using modified Fuzzy $\mathrm{C}$ means segmentation is done for accurate tumor shape extraction of malignant tumor and in feature extraction thresholding of output is carried out. Finally calculation of tumor shape, position, and stage is done using specific formulae. After that the experimental results are compared with other algorithms graphs will be generated. The proposed method will give more accurate result. Also 3D analysis will be done through 3D slicer. In future this system can be implemented with some other algorithm which will give more accuracy and save more time.

\section{ACKNOWLEDGMENT}

Purnita Majumder expresses her gratitude to Prof. V. P. Kshirsagar for his constant support and encouragement. He 
has been constant source of inspiration. She also thanks to Prof. M. B. Nagori, Professor, Computer Science \& Engineering Depratment, Government College of Engineering, Aurangabad, for her valuable and firm suggestions.

\section{REFERENCES}

[1] J.selvakumar, A.Lakshmi, T.Arivoli, "Brain tumor segmentation and its area calculation in brain MR images using K-mean clustering and fuzzy C-Mean algorithm", 2012.

[2] Alan Wee-Chung Liew, Member, IEEE, and Hong Yan, Senior Member, IEEE, "An Adaptive Spatial Fuzzy Clustering Algorithm for 3-D MR Image Segmentation", in IEEE transactions on medical imaging, Vol. 22, No 9, Sept 2003.

[3] Jamal Ghasemi, Reza Ghaderi, Mohamad Reza Karami mollaei, Ali Hojjatoleslami "Separation of Brain Tissues in MRI based on Multi-Dimensional FCM and Spatial Information", in FSKD-2011.

[4] Linju Lu, Min Li, Xiaoying Zhang “An Improved MR image segmentation method based on Fuzzy C-means Clustering", in IJCSET 2013.

[5] Zexuan Ji, Yong Xia, Quansen Sun, Qiang Chen, Deshen Xia, David Dagan Feng "Fuzzy Local Gaussian Mixture
Model for Brain MR Image Segmentation" in IEEE Transactions on Information Technology In Biomedicine, Vol. 16, No. 3, May 2012.

[6] Shally HR, Chitharanjan K,"Tumor volume calculation of brain from MRI slices", 2013.

[7] Mei Yeen Choong, Wei Yeang Kow, Yit Kwong Chin, Lorita Angeline, Kenneth Tze Kin Teo, "Image Segmentation via Normalised Cuts and Clustering Algorithm", in IEEE International Conference on Control System, Computing and Engineering, 2012.

[8] Tse-Wei Chen, Yi-Ling Chen , Shao-Yi Chien, "Fast Image Segmentation Based on K-Means Clustering with Histograms in HSV Color Space", Journal of Scientific Research ISSN I452-2I6X Vol. 44 No.2, 2010.

[9] Anil Z Chitade, "Colour based imagesegmentation using k-means clustering", in International Journal of Engineering Science and Technology, Vol. 2(10), 2010.

[10] T. Kanungo, D. M. Mount, N. Netanyahu, C. Piatko, R. Silverman, \& A. Y.Wu, "An efficient k-means clustering algorithm:Analysis and implementation", in Proc. IEEE Conf. Computer Vision and Pattern Recognition, 2002.

[11] A. Suman Tatiraju, "Image Segmentation using k-means clustering, EM and Nonnalized Cuts", Symposium of Discrete Algorithms, 2008. 